\title{
Major Depressive Disorder (MDD) and Antidepressant Medication Are Overrepresented in High-Dose Statin Treatment
}

\author{
Michael Leutner ${ }^{1}$, Caspar Matzhold ${ }^{2,3}$, Alexander Kautzky ${ }^{4}$, Michaela Kaleta ${ }^{2,3}$, \\ Stefan Thurner ${ }^{2,3,5,6}$, Peter Klimek ${ }^{2,3}$ and Alexandra Kautzky-Willer ${ }^{1,7 *}$ \\ ${ }^{1}$ Clinical Division of Endocrinology and Metabolism, Department of Internal Medicine III, Medical University of Vienna, Vienna, \\ Austria, ${ }^{2}$ Section for Science of Complex Systems, Center for Medical Statistics, Informatics, and Intelligent Systems \\ (CeMSIIS), Medical University of Vienna, Vienna, Austria, ${ }^{3}$ Complexity Science Hub Vienna, Vienna, Austria, ${ }^{4}$ Department of \\ Psychiatry and Psychotherapy, Medical University of Vienna, Vienna, Austria, ${ }^{5}$ Santa Fe Institute, Santa Fe, NM, \\ United States, ${ }^{6}$ Institute for Applied Systems Analysis (IIASA), Laxenburg, Austria, ${ }^{7}$ Gender Institute, Gars am Kamp, Austria
}

\section{OPEN ACCESS}

Edited by:

Claudia Penna,

University of Turin, Italy

Reviewed by:

Tianmei Si

Peking University Sixth Hospital, China

Tommaso Angelone,

University of Calabria, Italy

*Correspondence:

Alexandra Kautzky-Willer

alexandra.kautzky-

willer@meduniwien.ac.at

Specialty section:

This article was submitted to Family Medicine and Primary Care,

a section of the journal

Frontiers in Medicine

Received: 18 September 2020

Accepted: 12 January 2021

Published: 11 February 2021

Citation:

Leutner M, Matzhold C, Kautzky A, Kaleta M, Thurner S, Klimek $P$ and

Kautzky-Willer A (2021) Major

Depressive Disorder (MDD) and

Antidepressant Medication Are

Overrepresented in High-Dose Statin

Treatment. Front. Med. 8:608083.

doi: 10.3389/fmed.2021.608083
Objective: To examine the dose-dependent relationship of different types of statins with the occurrence of major depressive disorder (MDD) and prescription of antidepressant medication.

Methods: This cross-sectional study used medical claims data for the general Austrian population ( $n=7,481,168)$ to identify all statin-treated patients. We analyzed all patients with MDD undergoing statin treatment and calculated the average defined daily dose for six different types of statins. In a sub-analysis conducted independently of inpatient care, we investigated all patients on antidepressant medication (statin-treated patients: $n=98,913$; non-statin-treated patients: $n=789,683)$. Multivariate logistic regression analyses were conducted to calculate the risk of diagnosed MDD and prescription of antidepressant medication in patients treated with different types of statins and dosages compared to non-statin-treated patients.

Results: In this study, there was an overrepresentation of MDD in statin-treated patients when compared to non-statin-treated patients (OR: 1.22, 95\% Cl: 1.20-1.25). However, there was a dose dependent relationship between statins and diagnosis of MDD. Compared to controls, the ORs of MDD were lower for low-dose statin-treated patients (simvastatin $>0-<=10 \mathrm{mg}$ :OR: $0.59,95 \% \mathrm{Cl}$ : 0.54-0.64; atorvastatin $>0-<=10$ mg:OR:0.65, 95\%Cl: 0.59-0.70; rosuvastatin $>0-<=10 \mathrm{mg}: \mathrm{OR}: 0.68,95 \% \mathrm{Cl}$ : 0.53-0.85). In higher statin dosages there was an overrepresentation of MDD (simvastatin $>40-<=60$ mg:OR: 2.42 , 95\% Cl: 2.18-2.70, >60-80 mg:OR: 5.27, 95\% Cl: 4.21-6.60; atorvastatin $>40-<=60$ mg:OR: $2.71,95 \% \mathrm{Cl}: 1.98-3.72,>60-<=80$ mg:OR: 3.73, 95\% Cl: 2.22-6.28; rosuvastatin $>20-<=40 \mathrm{mg}:$ OR: $2.09,95 \% \mathrm{Cl}$ : 1.31-3.34). The results were confirmed in a sex-specific analysis and in a cohort of patients taking antidepressants, prescribed independently of inpatient care.

Conclusions: This study shows that it is important to carefully re-investigate the relationship between statins and MDD. High-dose statin treatment was related to an overrepresentation, low-dose statin treatment to an underrepresentation of MDD.

Keywords: statins, depression, dyslipidemia, dosage, precision medicine 


\section{INTRODUCTION}

Statins rank among the most-prescribed drugs worldwide and have significant lipid-lowering effects and hence they are usually prescribed to prevent and treat cardiovascular disease (CVD). Recently published guidelines of the European Society of Cardiology (ESC/EAS) recommend that high-risk patients, such as patients with type-2 diabetes with organ damage or patients with CVD, should have low-density lipoprotein cholesterol (LDL-cholesterol) levels lower than $55 \mathrm{mg} / \mathrm{dl}$ (1). The main mechanism of statins is inhibition of 3-hydroxy-3-methylglutaryl-coenenzyme A reductase (HMG-CoA reductase), which in turn inhibits synthesis of mevalonic acid, the main substrate for the synthesis of cholesterol. Cholesterol itself is the basic substance for the synthesis of essential hormones such as sex hormones or vitamin D. In this context, earlier studies have shown that statins can lower the concentrations of sex hormones for instance (2-5).

A connection between sex hormones and depression has also been demonstrated by earlier studies. Low levels of estrogen have been associated with depression in women (6-10) and withdrawal of hormone therapy for remitted perimenopausal depression has been linked to recurrence of depressive symptoms (7). Similar results have been shown for low testosterone levels in men (11-13). Earlier studies report associations between low levels of cholesterol and consequently statin usage and depression, as well as related symptoms such as lowered mood, aggression and suicidality (14-17). A potential mechanism for these associations is impaired serotonin signaling, as cholesterol is required for serotonin $1 \mathrm{~A}$ receptor function (18-20) and hence downregulation of the serotonin $1 \mathrm{~A}$ receptor has been linked to mood disorders such as depression (21).

More recently, the neuroinflammation hypothesis for depression has gained traction, also pointing to antidepressant effects of anti-inflammatory agents such as statins (22). Along these lines, some studies report a protective effect of statins on the development of depression (22-29). One of the major problems of the existing literature is that especially the relationship between high-dose statin treatment and MDD has yet to be investigated in detail. Data on the relationship between highdose statin treatment and MDD in randomized controlled trials (RCTs) are particularly sparse and hence the existing literature does not clearly demonstrate that statins have an antidepressant effect, which is the main reason why they are not considered in antidepressant therapy (30). Given the paucity of data, the aim of the present study was to investigate the relationship between statins of different types and dosages and MDD.

\section{METHODS}

\section{Study Design}

This cross-sectional retrospective analysis investigated medical claims data for the general Austrian population. Two groups [patients (1) with and (2) without statin treatment] were compared in order to investigate the relationship between statin treatment and MDD.

\section{Patient Population}

In the present analysis, health data were investigated for all Austrians receiving health care services, i.e., $\sim 97 \%$ of the population. These data include all diagnoses recorded during a hospital stay and data for all drug prescriptions exceeding a prescription charge of EUR 4.70. All patients alive during the observational period from January 2006 to December $2007(n=7,945,775)$ were analyzed, and age and sex were noted. Patients born in these years or aged $>90$ were excluded. The final study cohort consisted of $7,481,168$ patients (3,507,903 males; 3,973,265 females). Medical prescriptions during the study period were analyzed using the Anatomical Therapeutic Chemical (ATC) Classification System codes.

\section{Characterization of Patients With MDD}

We identified all patients diagnosed with MDD during hospital stays by using primary and secondary diagnoses, as defined by the International Classification of Diseases, 10th revision (ICD-10), codes. We classified patients as having MDD if they had a primary or secondary diagnosis of F32 (major depressive disorder, single episode) or F33 (major depressive disorder, recurrent). In order to strengthen our results, we performed a sub-analysis in which we investigated all patients who had been prescribed antidepressants $(n=888,596)$, irrespective of inpatient hospital stays. In another sub-analysis, we considered only patients with at least one hospital stay $(n=2,011,334)$, i.e., for whom diagnoses could in principle have been recorded.

\section{Characterization of Statin-Treated Patients and the Control Group}

The statin-treated group consisted of patients prescribed one of the following six statins in at least four different quarters of a year (representing the common prescription procedure in Austria and patients' compliance) during the observational period: simvastatin (ATC-code:C10AA01), lovastatin (ATCcode:C10AA02), pravastatin (ATC-code:C10AA03), fluvastatin (ATC-code:C10AA04), atorvastatin (ATC-code:C10AA05), and rosuvastatin (ATC-code:C10AA07). Patients who had been prescribed two different types of statins over the observational period of 2 years were excluded $(n=5,361)$. The control group (non-statin-treated patients) consisted of patients to whom no statins were dispensed during the observation period. Antidepressant use was measured as the dispensing of at least one antidepressant (ATC code starting with N06A) or in combination with psycholeptics (N06CA), olanzapine (N05AH03), quetiapine (N05AH04), sulpiride (N05AL01), lithium (N05AN01), or benzodiazepine derivatives (N05BA) during the observation period.

Finally, the following groups were defined and compared:

1) Statin-treated patients vs. non-statin-treated patients.

2) Sub-analysis (independent of inpatient care): statin-treated patients on antidepressant medication vs. non-statin-treated patients on antidepressant medication. 


\section{Average Daily Doses}

Average daily doses for the drugs were calculated from the prescribed dosage, which was converted from defined daily dose to $\mathrm{mg}$ and divided by the number of days that were not spent in a hospital. To obtain the individual averages of the daily doses, we extracted the individual drug histories, including information on dates of received prescriptions and the corresponding dosage of the prescribed statin. The average was calculated by dividing the sum of all amounts of the administered drug by the sum of treatment days, minus the days a patient spent in hospital. The hospital days were subtracted on the assumption that the patients were treated with statins during the hospital stay.

In order to ensure precise characterization and interpretation of each substance, we defined groups according to the average daily dose for each statin, resulting in the following categories: $>0-10 \mathrm{mg},>10-20 \mathrm{mg},>20-40 \mathrm{mg},>40-60 \mathrm{mg},>60-80 \mathrm{mg}$.

\section{Ethical Approval}

This study has been approved by the ethics commission of the Medical University of Vienna (EK-Nr.: 1020/2020). A detailed statement on ethical approval is provided in the Supplementary Material. Written informed consent from the patients was not required to participate in this study in accordance with the national legislation and the institutional requirements.

\section{Statistical Analysis}

We calculated odds ratios (ORs) between each case of statin use and diagnosis of MDD in a matched cohort analysis (each statintreated patient was matched to three members of the control group of the same age and sex). Weighted multiple logistic regression, as described in similar analysis designs (31), was used to investigate this association while controlling for age, sex, dosage, and prescription of other medications (for diabetes and fibrates).

Patients were assigned a categorical variable for each statin comprising the respective average daily dose in $\mathrm{mg}$. We controlled for use of other medications (at least four different quarters during which 20 different glucose-lowering drugs were dispensed, including metformin; three fibrates) by introducing binary dummy variables. Model quality was evaluated using the adjusted R-squared statistic, multi-collinearity via the variance inflation factor (VIF). Stratification was used to control for other diagnoses potentially associated with statin use and MDD and which could in principle act as confounding factors. These robustness tests excluded all patients with a diagnosis of ischaemic heart diseases (any code from the range I20I25), diseases of the arteries, including arterioles and capillaries (I70-I79), stroke (I63-I64), diabetes (E10-E11), obesity (E66), hypothyroidism (E02-E03), cancer (any code from the range C00-C97), dementia in Alzheimer's disease (specific ICD code for patients with Alzheimer's disease additionally diagnosed with dementia) and Alzheimer's disease (F00, G30), pain (R52), and sleep disorder (G47). Statistical analysis was performed using standard packages of Matlab.

\section{RESULTS}

Of a total of $7,481,168$ patients, 84,638 (1.13\% of the general Austrian population) were diagnosed with MDD during a hospital stay and 888,596 (11.88\% of the general Austrian population) were undergoing antidepressant therapy (prescribed independently of a hospital stay). The baseline characteristics of statin-treated patients and their age- and sex-matched control group are described in Table 1. In general, statin-treated patients (males and females) were more likely to have been diagnosed with MDD and were more commonly treated with antidepressants when compared to age- and sex-matched non-statin treated patients (OR: 1.54, CI: 1.53-1.56).

Supplementary Table 1 shows the baseline characteristics of the statin-treated patients with diagnosed MDD in comparison to patients with MDD without statin treatment. Depressed patients undergoing statin treatment often received antidepressant and antidiabetic therapy and displayed a higher prevalence rate of CVD, stroke, diseases of the arteries, overweight, obesity and hypothyroidism than those without statin treatment.

\section{Dose-Dependence of Statins on Diagnosis of MDD}

In the general population there was an increased risk of diagnosis with depression in statin-treated patients when compared to matched controls (OR: 1.22, CI: 1.20-1.25). Further, there was a potency- and dose-dependent relationship between statins and diagnosis of MDD (see Table 2, Figure 1, Supplementary Figures 2, 3 for multiple logistic regression analyses and odds of diagnosis). In comparison to non-statintreated patients, there was an underrepresentation of diagnosed MDD in patients receiving lovastatin in doses of $>0-20 \mathrm{mg}$ (0-10 mg: OR: 0.12, 95\% CI: 0.05-0.27; 10-20 mg: OR: 0.62, 95\% CI: 0.41-0.95). Similar results were observed for pravastatin $>0-20 \mathrm{mg}$ (0-10 mg: OR: 0.36, 95\% CI: 0.29-0.44; 10-20 mg: OR: 0.63 , CI: $0.56-0.70)$, simvastatin $>0-20 \mathrm{mg}(0-10 \mathrm{mg}$ : OR: 0.59 , 95\% CI: $0.54-0.64$; $10-20 \mathrm{mg}$ : OR: 0.81 , CI: $0.77-$ 0.86 ), atorvastatin $>0-10 \mathrm{mg}$ (OR: $0.65,95 \% \mathrm{CI}: 0.59-0.70$ ), rosuvastatin $>0-10 \mathrm{mg}$ (OR: $0.68,95 \% \mathrm{CI}: 0.53-0.85$ ) and fluvastatin $>10-60 \mathrm{mg}$. The results in Table 2 also demonstrate that the lower risk of diagnosis with MDD in low-dose statintreated patients decreased with an increase in the potencies of statins. The OR increased with potency, the lowest OR beginning in lovastatin-treated patients. Although there was an underrepresentation of diagnosed MDD in low-dose statin treatment, the higher dosages showed an overrepresentation of MDD. Dosages of $>20 \mathrm{mg}$ of simvastatin (20-40 mg: OR: 1.28 , 95\% CI: $1.21-1.35$; 40-60 mg: OR: 2.42 , 95\% CI: $2.18-2.70$; 60-80 mg: OR: $5.27,95 \% \mathrm{CI}: 4.21-6.60),>10 \mathrm{mg}$ atorvastatin (10-20 mg: OR: 1.19, 95\% CI: 1.06-1.33; 20-40 mg: OR: 1.91, 95\% CI: 1.60-2.27;40-60 mg: OR: 2.71, 95\% CI: 1.98-3.72; 6080 mg: OR: $3.73,95 \% \mathrm{CI}: 2.22-6.28)$ and rosuvastatin $>20 \mathrm{mg}$ (20-40 mg: OR: 2.09, 95\% CI: $1.31-3.34)$ were related to an overrepresentation of MDD when compared to controls without statin treatment. 
TABLE 1 | Baseline characteristics of the study population, showing group size, age and the absolute and relative frequencies of depression, use of other medications (insulin, oral antidiabetics, antidepressants) and comorbid conditions for males and females in the statin-treated and in the matched control group.

\begin{tabular}{|c|c|c|c|c|}
\hline & \multicolumn{2}{|c|}{ Statin-treated patients } & \multicolumn{2}{|c|}{ Non-statin-treated patients } \\
\hline Age (mean +/- SD) & $64.91+/-10.86$ & $68.88+/-10.41$ & $64.91+/-10.86$ & $68.88+/-10.41$ \\
\hline Depression (F32-F33) & $2,947^{\star}(1.76 \%)$ & $6,774^{\star}(3.98 \%)$ & 6,907 (1.38\%) & $15,953(3.12 \%)$ \\
\hline Antidepressants & $35,379^{\star}(21.19 \%)$ & $63,534^{\star}(37.32 \%)$ & $73,675(14.71 \%)$ & $140,846(27.57 \%)$ \\
\hline CVD (I20-I25) & $32,611^{\star}(19.53 \%)$ & $20,803^{*}(12.22 \%)$ & $21,733(4.34 \%)$ & $19,631(3.84 \%)$ \\
\hline Stroke $(163,164)$ & $4,367^{\star}(2.62 \%)$ & $3,684^{\star}(2.16 \%)$ & $5,826(1.16 \%)$ & $5,860(1.15 \%)$ \\
\hline Diseases of arteries (170-179) & $10,552^{*}(6.32 \%)$ & $7,569^{\star}(4.45 \%)$ & 13,227 (2.64\%) & $10,415(2.04 \%)$ \\
\hline Overweight and obesity (E66) & $7,164^{\star}(4.29 \%)$ & $7,149 *(4.20 \%)$ & $8,384(1.67 \%)$ & $11,061(2.17 \%)$ \\
\hline Hypothyroidism (E02, E03) & $1,239^{\star}(0.74 \%)$ & $3,277^{\star}(1.92 \%)$ & $2,551(0.51 \%)$ & $6,735(1.32 \%)$ \\
\hline
\end{tabular}

SD, standard deviation; CVD, cardiovascular disease, Asterisks denote statistically significant differences between statin- and non-statin treated patients; ${ }^{\star *} p<0.01$, ${ }^{\star} p<0.05$.

TABLE 2 | Dose-dependent relationship between statins and diagnosis of depression.

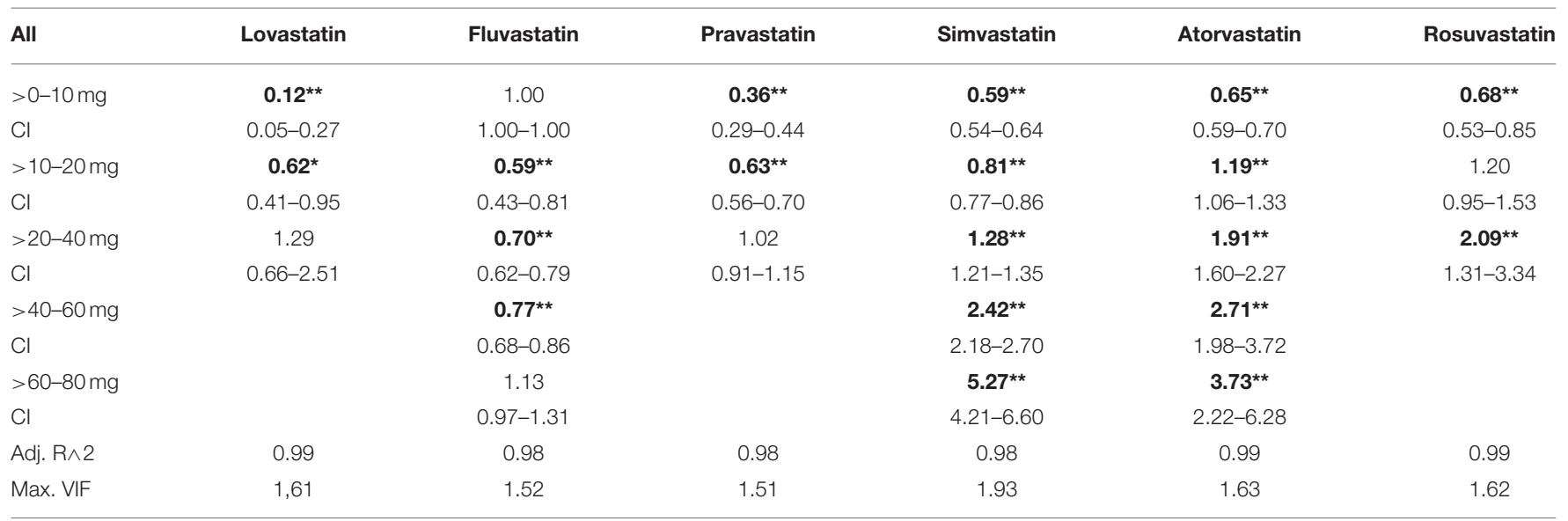

${ }^{* *} p<0.01 ;{ }^{*} p<0.05$

Bold values represent the significant ORs.

\section{Robustness Test for Diseases Commonly Related to MDD}

We further conducted a robustness test to estimate the influence of diseases directly related to MDD, in particular ischemic heart disease, diseases of arteries, stroke, diabetes, obesity, hypothyroidism, cancer, dementia in Alzheimer's disease, Alzheimer's disease, pain, and sleep disorders. We thus tested whether the dosage-dependent MDD risk trajectories are independent of the above-mentioned disease groups (see Figure 2, Supplementary Material: baseline tests and Supplementary Figures 5-10). In these robustness tests the results showed that the observed dose dependencies followed the same trend, with an underrepresentation of diagnosed MDD in low-dose and an overrepresentation in high-dose statin-treatment. The dose-dependent relationship could also be observed in a sub-analysis that only included patients hospitalized at least once (see Supplementary Figure 1).

\section{Sex-Specific Analysis of the Dose-Dependent Relationship Between Statins and Diagnosis of MDD}

In both male and female patients, the results shown in the general population were confirmed, demonstrating that lowdose statin treatment is related to underrepresentation of diagnosed MDD whereas high-dose statin treatment is related to overrepresentation when compared to non-statin-treated patients. Especially for high-dose atorvastatin treatment, we 


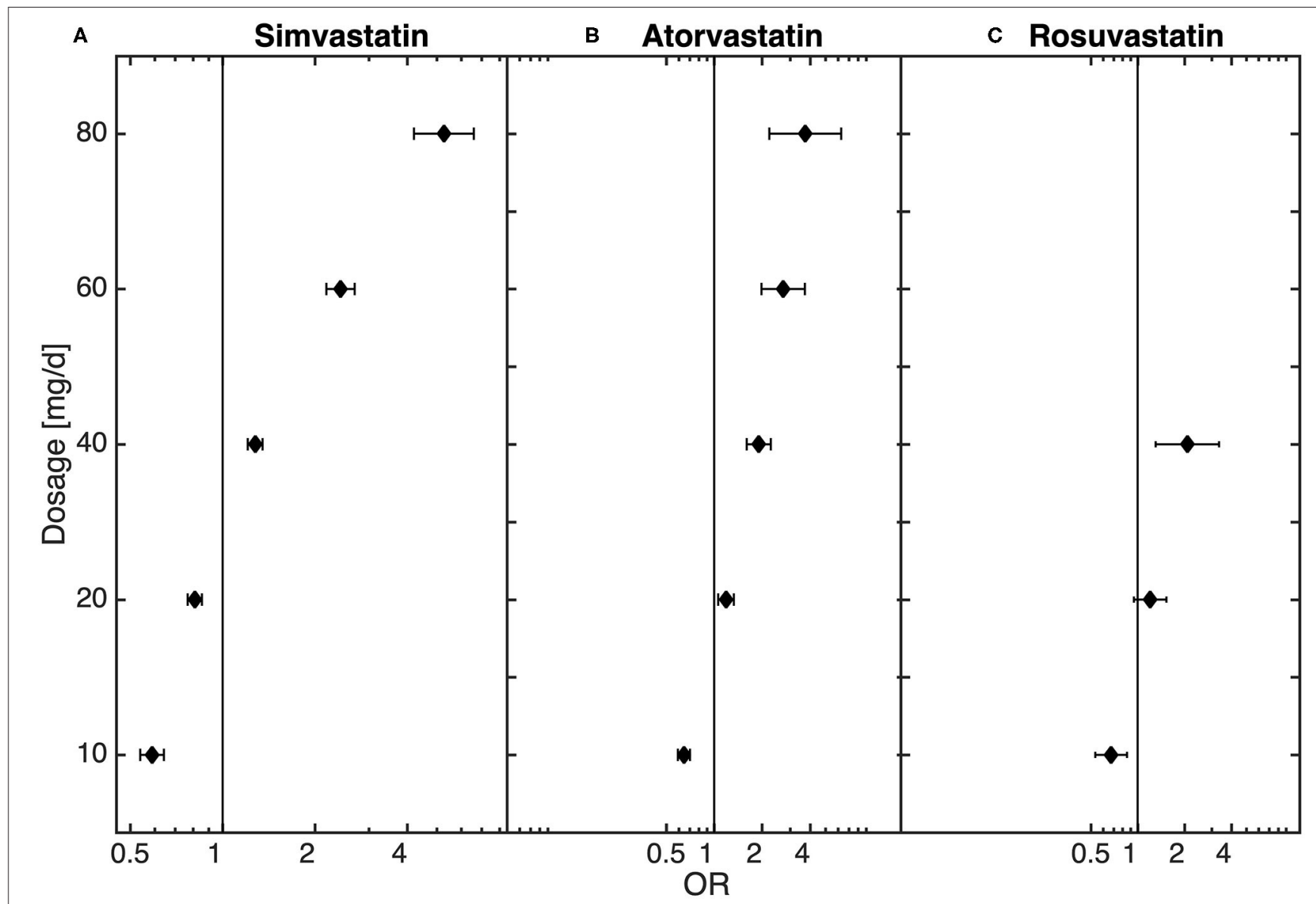

FIGURE 1 | Dose-dependent relationship between (A) simvastatin ( $n=214,021)$, (B) atorvastatin $(n=37,919),(\mathbf{C})$ and rosuvastatin $(n=8,209)$ and diagnosis of depression obtained from the logistic regression model.

found that the risk of diagnosis with MDD in females was nearly double than in males. Further details can be found in Supplementary Tables 2, 3, Supplementary Figure 4.

\section{Sub-Analysis-Dose-Dependent Relationship Between Statin Treatment and Antidepressant Therapy}

The final sub-analysis included 888,596 patients receiving antidepressant medication prescribed independently of hospitalization. Statin treatment was recorded for 98,913 of these patients. In the sub-analysis of all patients treated with antidepressants, similar dose-dependent results in statin-treated patients could be observed. Thus, low-dose statin treatment was related to an underrepresentation and high-dose statin treatment to an overrepresentation of antidepressant medication when compared to non-statin-treated patients (Figure 3).

\section{DISCUSSION}

In the present study, we investigated the relationship between different types and dosages of statins and diagnosis of MDD in comparison to non-statin-treated patients. We were able to demonstrate that there was an increased risk of diagnosed MDD in patients treated with higher doses of statins when compared to non-statin-treated patients. Interestingly, low-dose statin treatment was related to an underrepresentation of MDD when compared to non-statin-treated patients. Our findings are also supported by sex-specific results and displayed no qualitative change after exclusion of patients with diseases closely related to diagnosis of MDD, such as cardiovascular disease or diabetes, and to statin use. The dose-dependent results were also observed in a sub-analysis only including patients taking antidepressant medication prescribed independently of hospitalization.

Given the physiological mechanisms associated with statins, a relationship between statin treatment and mood disorders such as MDD seems plausible. Statins inhibit HMG-CoA reductase, which is the main mechanism for the synthesis of cholesterol, resulting in lower cholesterol levels (32) and consequently subsequent products such as sex hormones (2-5). Close relationships between low sex hormone levels and mood disorders were also suggested $(7,9,11,12)$. We have recently published evidence for a dose-dependent increased risk of diagnosis of osteoporosis in statin-treated patients and 


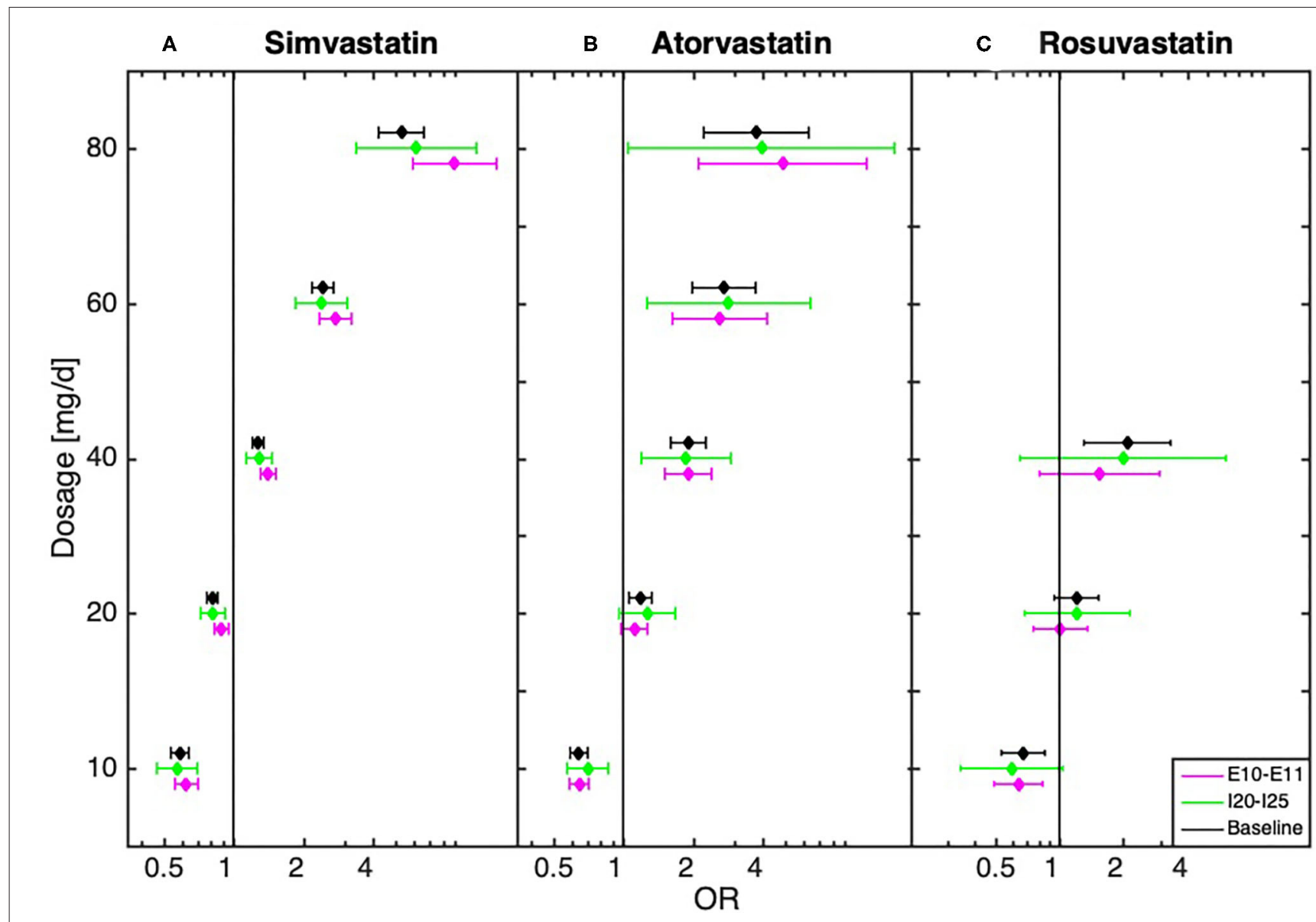

FIGURE 2 | Dose-dependent relationship between (A) simvastatin, (B) atorvastatin, and (C) rosuvastatin and diagnosis of depression for baseline (all statin-treated patients) and after the exclusion of patients with cardiovascular disease (I20-I25, $n=53,414)$ and $(n=71,722) /$ or diabetes (E10-E11, $n=32,815)$ obtained from the logistic regression model.

hypothesized that specifically higher dosages could inhibit the synthesis of sex hormones on a clinically relevant level and therefore advance bone resorption (33). By showing that there is also a dose-dependent relationship with diagnosed MDD, here we demonstrate for the first time that it is important to consider the different types of statins and their dosages when investigating the relationship between statin treatment and diagnosis of MDD.

Concerns about potential central nervous side-effects of statins have been raised for almost 30 years. A series of pioneering work made a case for increased depression rates as well as non-cardiovascular mortality due to violent incidents and suicide in patients with low cholesterol and thus in statin users (34-39). Interestingly, these early positive associations were rarely replicated (17), as more recent large clinical and register-based studies have generally implied no increased risk of developing MDD in statin users (22). On the contrary, protective effects have been reported and were related to neuroinflammation as a potential key mechanism of depression (40-42). Nevertheless, overall the mechanisms of putative association in either direction are insufficiently understood. Most importantly, the majority of studies have neglected different potencies and dosages of statins despite recent advances linking statins with high lipophilia and hence permeability of the blood-brain barrier but not establishing a connection between hydrophilic statins and MDD (43). Hence it is not clear whether under high-dose statin therapy a stronger downregulation of sex hormones, which are closely related to MDD, could possibly overrule the positive anti-inflammatory effect of statins on MDD.

Data regarding the dose-dependent risk of MDD in statintreated patients are particularly sparse. For instance, in placebocontrolled clinical trials it has been shown that both lowdose lovastatin treatment ( $30 \mathrm{mg})(44)$ and low-dose simvastatin treatment $(20 \mathrm{mg})(45,46)$ resulted in significant relief of depressive symptoms. There are also other prospective studies demonstrating that low-dose statin treatment, such as $20 \mathrm{mg}$ of atorvastatin $(46,47)$, could have positive effects on symptoms of depression. These results indicate that low-dose statin treatment could indeed be effective in the treatment of depression and are consequently in accordance with our results, as we found an underrepresentation of MDD in patients treated with lower doses of lovastatin (0-20 mg), pravastatin (0-20 mg), simvastatin $(0-20 \mathrm{mg})$, atorvastatin $(0-10 \mathrm{mg})$, and rosuvastatin $(0-10 \mathrm{mg})$. 


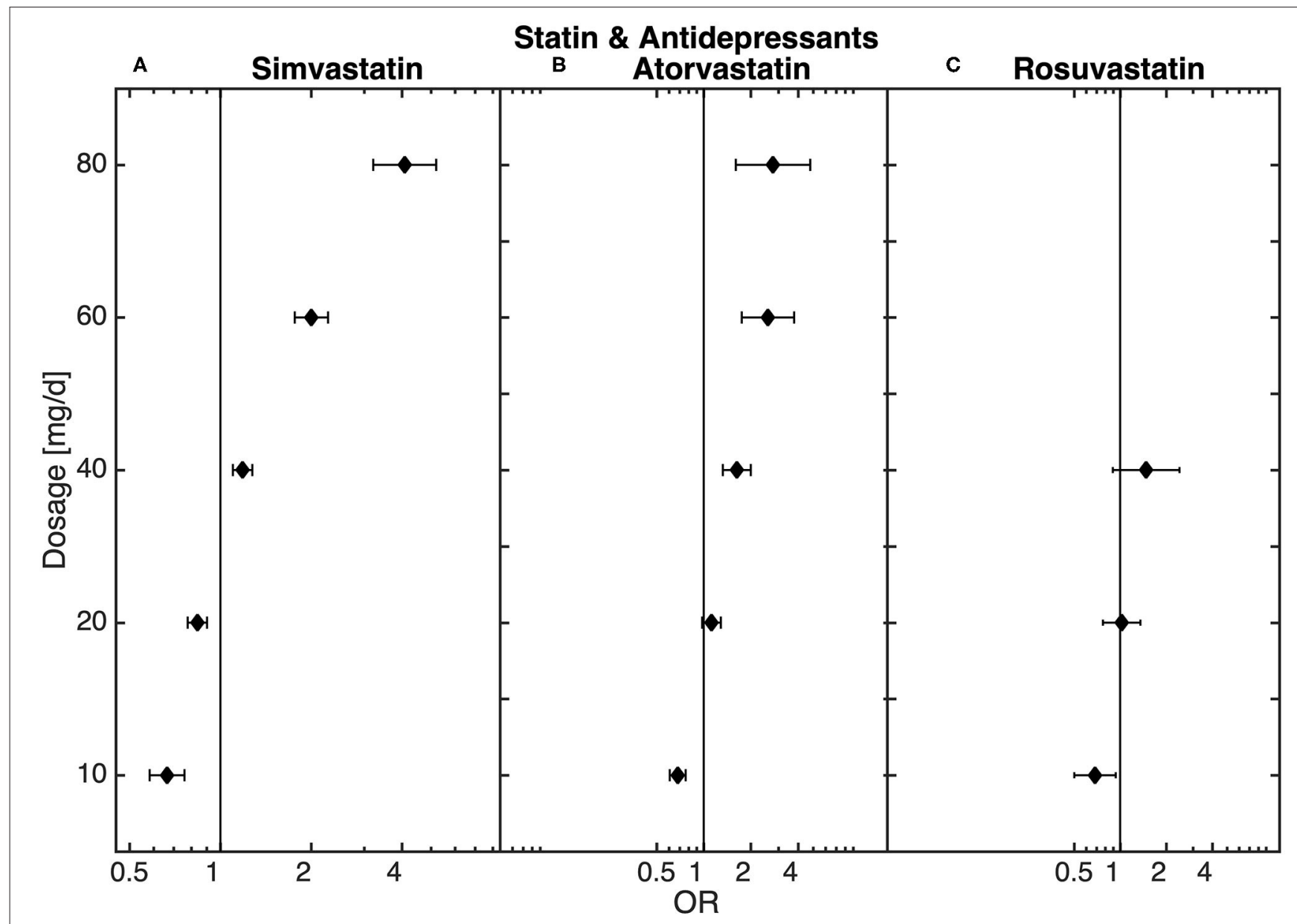

FIGURE 3 | Dose-dependent relationship between (A) simvastatin, (B) atorvastatin, and (C) rosuvastatin and antidepressant medication $(n=98,913)$ obtained from the logistic regression model.

There is evidence that statins can have positive effects on depression via an anti-inflammatory effect, modulation of cytokines and reduction of oxidative stress. Improved quality of life due to improved health consciousness and treatment compliance and reduced cardiovascular risk has also been linked to a potential antidepressant effect of statins (48). Nevertheless, especially the relationship to higher dosages has yet to be investigated sufficiently. That it is also important to investigate the different types of statins with their different potencies has been demonstrated by a Swedish national cohort study showing that simvastatin had a protective effect on depression, whereas atorvastatin treatment increased the risk. However, there was no detailed investigation of different dosages (25).

In the present study, higher dosages were related to an overrepresentation of MDD in statin-treated patients. These results could be confirmed by a sex-specific analysis and remain unchanged after exclusion of patients with diseases closely related to MDD. Further, similar results were observed in a sub-analysis investigating all patients taking antidepressant medication prescribed independently of hospitalization. Thus, dosage and potency may be the deciding factors for protective or risk-increasing effects. Whether a possible downregulation of hormones directly related to MDD under high-dose statin treatment could overwhelm the described positive antiinflammatory effects of statins on MDD should be investigated in larger prospective clinical trials. The overrepresentation of diagnosed MDD in high-dose statin-treated patients is of special interest with regard to the synthesis and processing of cholesterol to essential hormones such as steroid hormones or Vitamin D. A study by Chan et al. investigated the effect of high-dose $80 \mathrm{mg}$ simvastatin therapy on mood in a cohort of 140 patients with secondary progressive multiple sclerosis by conducting a 24 month, double-blind controlled trial. They showed similar results to those of our study, namely that high-dose statin treatment was related to increased severity of depressive symptoms, as measured using the Hamilton Depression Rating Scale (HAM-D) (49). Hence the fact that cholesterol is required for the serotonin 1A receptor to function (18-20) also has to be considered, since down-regulation of this receptor is closely related to mood disorders (21).

Possible interactions with the metabolization of statins should not be discounted either, as in our study females were at over double the risk of diagnosis with MDD than males when receiving high-dose atorvastatin treatment. One thus has to 
consider that atorvastatin is metabolized by cytochrome $\mathrm{P} 450$ 3A4 (CYP3A4), which is also involved in the metabolism of estrogens.

Our study has both limitations and strengths. First, registerbased studies are limited by the fact that there is no opportunity to precisely characterize the diagnosis of the diseases using clinical data, since we only have access to ICD codes. In the present study, the number of patients diagnosed with depression was in relation to the actual literary lower. However, a strength is that antidepressant medication was also recorded independently of hospital stays and this number reflects the actual literary and might compensate underreporting in the second group. A further limitation of the present study is that we do not have information on the duration of statin treatment and that we cannot infer causal effects using the cross-sectional study design. Additionally, it is not possible to screen the data for potential associations between different time periods of statin treatment and the relationship to major depressive disorders and/or antidepressant medication. Hence especially high-dose statin-treated patients often had a history of cardiovascular events and a higher occurrence of cardiovascular risk markers and thus MDD could therefore be a consequence of CVD $(50,51)$. In our dataset from 2006 to 2007 we had no access to cholesterol levels and could therefore only hypothesize that there could be a dose dependent relationship between statins and the upcoming synthesis of vital hormones (e.g., testosterone and estrogen), which are directly related to MDD. Additionally, we have no information on marital and socioeconomic status, which is also related to MDD. Also, we have no information about common side effects of statins, such as muscle symptoms or reduced exercise tolerance, and it is known that these symptoms may induce mood disorders. Further, it was not possible to provide detailed analysis of treatment adherence. In comparison to controls, statin-treated patients are characterized in the present study by a higher rate of comorbidities and it is a well-known fact that diseases such as diabetes mellitus or $\mathrm{CHD}$, for instance, are closely related to the development of MDD. However, in our robustness tests, there was no qualitative change in the results after the exclusion of patients with such diagnoses.

\section{CONCLUSION}

In conclusion, our results demonstrate that there exists a dose-dependent relationship between statins and diagnosis of MDD, substantiating both underrepresentation of MDD in low-dose statin treatment and increased risk of diagnosis with MDD in high-dose treatment. Considering the widespread use

\section{REFERENCES}

1. Mach F, Baigent C, Catapano AL, Koskinas KC, Casula M, Badimon L, et al. 2019 ESC/EAS Guidelines for the management of dyslipidaemias: lipid modification to reduce cardiovascular risk. Eur Heart J. (2019) 41:111-88. doi: 10.15829/1560-4071-2020-3826

2. Schooling CM, Au Yeung SL, Freeman G, Cowling BJ. The effect of statins on testosterone in men and women, a systematic review of statins primarily for disease prevention and increasingly stricter recommendations for tolerated cholesterol levels, these findings may be highly relevant for clinical routine across a broad spectrum of medical disciplines. This is an important and interesting approach for precision medicine in particular. Nevertheless, keeping in mind the limitations of registerbased studies, prospective and longitudinal trials are urgently needed to validate our findings and further elucidate the mechanisms involved.

\section{DATA AVAILABILITY STATEMENT}

The data is not available to access because this is a consolidated research database that is only accessible for selected partners under a strict data protection policy.

\section{ETHICS STATEMENT}

The studies involving human participants were reviewed and approved by Medical University of Vienna. Written informed consent for participation was not required for this study in accordance with the national legislation and the institutional requirements.

\section{AUTHOR CONTRIBUTIONS}

ML, CM, AK, MK, ST, PK, and AK-W: study design. ML, CM, and PK: data analysis. ML and CM: manuscript writing. AK-W: is the guarantor of this work. All authors read, reviewed, and approved the final manuscript.

\section{FUNDING}

This study was funded by the Vienna Science and Technology Fund (MA16-045).

\section{ACKNOWLEDGMENTS}

We extend our thanks to Dr. Gottfried Endel for his assistance with data assessment.

\section{SUPPLEMENTARY MATERIAL}

The Supplementary Material for this article can be found online at: https://www.frontiersin.org/articles/10.3389/fmed. 2021.608083/full\#supplementary-material and meta-analysis of randomized controlled trials. BMC Med. (2013) 11:57. doi: 10.1186/1741-7015-11-57

3. Stanworth RD, Kapoor D, Channer KS, Jones TH. Statin therapy is associated with lower total but not bioavailable or free testosterone in men with type 2 diabetes. Diabetes Care. (2009) 32:541-6. doi: 10.2337/dc0 8-1183

4. Corona G, Boddi V, Balercia G, Rastrelli G, De Vita G, Sforza A, et al. The effect of statin therapy on testosterone levels in 
subjects consulting for erectile dysfunction. J Sex Med. (2010) 7(Pt.1):1547-56. doi: 10.1111/j.1743-6109.2009.01698.x

5. Zhang X, Li J, Zhou X, Guan Q, Zhao J, Gao L, et al. Simvastatin decreases sex hormone levels in male rats. Endocr Pract. (2017) 23:175-81. doi: 10.4158/EP161274.OR

6. Newhouse P, Albert K. Estrogen, stress, and depression: a neurocognitive model. JAMA Psychiatry. (2015) 72:727-9. doi: 10.1001/jamapsychiatry.2015.0487

7. Schmidt PJ, Ben Dor R, Martinez PE, Guerrieri GM, Harsh VL, Thompson $\mathrm{K}$, et al. Effects of estradiol withdrawal on mood in women with past perimenopausal depression: a randomized clinical trial. JAMA Psychiatry. (2015) 72:714-26. doi: 10.1001/jamapsychiatry.2015.0111

8. Xu Y, Sheng $\mathrm{H}$, Bao Q, Wang Y, Lu J, Ni X. NLRP3 inflammasome activation mediates estrogen deficiency-induced depression- and anxiety-like behavior and hippocampal inflammation in mice. Brain Behav Immun. (2016) 56:175-86. doi: 10.1016/j.bbi.2016.02.022

9. Almeida OP, Lautenschlager N, Vasikaram S, Leedman P, Flicker L. Association between physiological serum concentration of estrogen and the mental health of community-dwelling postmenopausal women age 70 years and over. Am J Geriatr Psychiatry. (2005) 13:142-9. doi: 10.1097/00019442-200502000-00008

10. Holsen LM, Spaeth SB, Lee JH, Ogden LA, Klibanski A, Whitfield-Gabrieli $\mathrm{S}$, et al. Stress response circuitry hypoactivation related to hormonal dysfunction in women with major depression. J Affect Disord. (2011) 131:379-87. doi: 10.1016/j.jad.2010.11.024

11. Shores MM, Sloan KL, Matsumoto AM, Moceri VM, Felker B, Kivlahan DR. Increased incidence of diagnosed depressive illness in hypogonadal older men. Arch Gen Psychiatry. (2004) 61:162-7. doi: 10.1001/archpsyc.61.2.162

12. Zarrouf FA, Artz S, Griffith J, Sirbu C, Kommor M. Testosterone and depression: systematic review and meta-analysis. J Psychiatr Pract. (2009) 15:289-305. doi: 10.1097/01.pra.0000358315.88931.fc

13. McHenry J, Carrier N, Hull E, Kabbaj M. Sex differences in anxiety and depression: role of testosterone. Front Neuroendocrinol. (2014) 35:42-57. doi: 10.1016/j.yfrne.2013.09.001

14. Steegmans PH, Hoes AW, Bak AA, van der Does E, Grobbee DE. Higher prevalence of depressive symptoms in middle-aged men with low serum cholesterol levels. Psychosom Med. (2000) 62:205-11. doi: 10.1097/00006842-200003000-00009

15. Tomson-Johanson K, Harro J. Low cholesterol, impulsivity and violence revisited. Curr Opin Endocrinol Diabetes Obes. (2018) 25:103-7. doi: 10.1097/MED.0000000000000395

16. Morales K, Wittink M, Datto C, DiFilippo S, Cary M, TenHave T, et al. Simvastatin causes changes in affective processes in elderly volunteers. $J$ Am Geriatr Soc. (2006) 54:70-6. doi: 10.1111/j.1532-5415.2005.00542.x

17. Hyyppa MT, Kronholm E, Virtanen A, Leino A, Jula A. Does simvastatin affect mood and steroid hormone levels in hypercholesterolemic men? A randomized double-blind trial. Psychoneuroendocrinology. (2003) 28:181-94. doi: 10.1016/S0306-4530(02)00014-8

18. Jafurulla M, Chattopadhyay A. Membrane lipids in the function of serotonin and adrenergic receptors. Curr Med Chem. (2013) 20:47-55. doi: 10.2174/0929867311302010006

19. Jafurulla M, Rao BD, Sreedevi S, Ruysschaert JM, Covey DF, Chattopadhyay A. Stereospecific requirement of cholesterol in the function of the serotonin1A receptor. Biochim Biophys Acta. (2014) 1838(Pt. B):158-63. doi: 10.1016/j.bbamem.2013.08.015

20. Pucadyil TJ, Chattopadhyay A. Role of cholesterol in the function and organization of G-protein coupled receptors. Prog Lipid Res. (2006) 45:295-333. doi: 10.1016/j.plipres.2006.02.002

21. Wang L, Zhou C, Zhu D, Wang X, Fang L, Zhong J, et al. Serotonin$1 \mathrm{~A}$ receptor alterations in depression: a meta-analysis of molecular imaging studies. BMC Psychiatry. (2016) 16:319. doi: 10.1186/s12888-016-1025-0

22. Salagre E, Fernandes BS, Dodd S, Brownstein DJ, Berk M. Statins for the treatment of depression: a meta-analysis of randomized, double-blind, placebo-controlled trials. J Affect Disord. (2016) 200:235-42. doi: 10.1016/j.jad.2016.04.047

23. Chuang CS, Yang TY, Muo $\mathrm{CH}$, Su HL, Sung FC, Kao $\mathrm{CH}$. Hyperlipidemia, statin use and the risk of developing depression: a nationwide retrospective cohort study. Gen Hosp Psychiatry. (2014) 36:497-501. doi: 10.1016/j.genhosppsych.2014.05.008

24. Kim SW, Bae KY, Kim JM, Shin IS, Hong YJ, Ahn Y, et al. The use of statins for the treatment of depression in patients with acute coronary syndrome. Transl Psychiatry. (2015) 5:e620. doi: 10.1038/tp.2015.116

25. Redlich C, Berk M, Williams LJ, Sundquist J, Sundquist K, Li X. Statin use and risk of depression: a Swedish national cohort study. BMC Psychiatry. (2014) 14:348. doi: 10.1186/s12888-014-0348-y

26. Yang CC, Jick SS, Jick H. Lipid-lowering drugs and the risk of depression and suicidal behavior. Arch Intern Med. (2003) 163:1926-32. doi: 10.1001/archinte.163.16.1926

27. O’Neil A, Sanna L, Redlich C, Sanderson K, Jacka F, Williams LJ, et al. The impact of statins on psychological wellbeing: a systematic review and meta-analysis. BMC Med. (2012) 10:154. doi: 10.1186/1741-7015-10-154

28. Parsaik AK, Singh B, Murad MH, Singh K, Mascarenhas SS, Williams MD, et al. Statins use and risk of depression: a systematic review and meta-analysis. J Affect Disord. (2014) 160:62-7. doi: 10.1016/j.jad.2013.11.026

29. Stafford L, Berk M. The use of statins after a cardiac intervention is associated with reduced risk of subsequent depression: proof of concept for the inflammatory and oxidative hypotheses of depression? J Clin Psychiatry. (2011) 72:1229-35. doi: 10.4088/JCP.09m05825blu

30. Kohler-Forsberg O, Otte C, Gold SM, Ostergaard SD. Statins in the treatment of depression: hype or hope? Pharmacol Ther. (2020) 215:107625. doi: 10.1016/j.pharmthera.2020.107625

31. Kautzky-Willer A, Thurner S, Klimek P. Use of statins offsets insulin-related cancer risk. J Intern Med. (2017) 281:206-16. doi: 10.1111/joim.12567

32. Istvan ES, Deisenhofer J. Structural mechanism for statin inhibition of HMGCoA reductase. Science. (2001) 292:1160-4. doi: 10.1126/science.1059344

33. Leutner M, Matzhold C, Bellach L, Deischinger C, Harreiter J, Thurner S, et al. Diagnosis of osteoporosis in statin-treated patients is dose-dependent. Ann Rheum Dis. (2019) 78:1706-11. doi: 10.1136/annrheumdis-2019-215714

34. Muldoon MF, Manuck SB, Matthews KA. Lowering cholesterol concentrations and mortality: a quantitative review of primary prevention trials. BMJ. (1990) 301:309-14. doi: 10.1136/bmj.301.6747.309

35. Law MR, Thompson SG, Wald NJ. Assessing possible hazards of reducing serum cholesterol. BMJ. (1994) 308:373-9. doi: 10.1136/bmj.308.6925.373

36. Boumendil E, Tubert-Bitter P. Depression-induced absenteeism in relation to antihyperlipidemic treatment: a study using GAZEL cohort data. Epidemiology. (1995) 6:322-5. doi: 10.1097/00001648-199505000-00023

37. Ploeckinger B, Dantendorfer K, Ulm M, Baischer W, Derfler K, Musalek $\mathrm{M}$, et al. Rapid decrease of serum cholesterol concentration and postpartum depression. BMJ. (1996) 313:664. doi: 10.1136/bmj.313.7058.664

38. Jacobs D, Blackburn H, Higgins M, Reed D, Iso H, McMillan G, et al. Report of the conference on low blood cholesterol: mortality associations. Circulation. (1992) 86:1046-60. doi: 10.1161/01.CIR.86.3.1046

39. Smith GD, Song F, Sheldon TA. Cholesterol lowering and mortality: the importance of considering initial level of risk. BMJ. (1993) 306:1367-73. doi: 10.1136/bmj.306.6889.1367

40. Kohler O, Krogh J, Mors O, Benros ME. Inflammation in depression and the potential for anti-inflammatory treatment. Curr Neuropharmacol. (2016) 14:732-42. doi: 10.2174/1570159X14666151208113700

41. Benros ME, Waltoft BL, Nordentoft M, Ostergaard SD, Eaton WW, Krogh J, et al. Autoimmune diseases and severe infections as risk factors for mood disorders: a nationwide study. JAMA Psychiatry. (2013) 70:812-20. doi: 10.1001/jamapsychiatry.2013.1111

42. Kohler $\mathrm{O}$, Benros ME, Nordentoft $M$, Farkouh ME, Iyengar RL, Mors $\mathrm{O}$, et al. Effect of anti-inflammatory treatment on depression, depressive symptoms, and adverse effects: a systematic review and meta-analysis of randomized clinical trials. JAMA Psychiatry. (2014) 71:1381-91. doi: 10.1001/jamapsychiatry.2014.1611

43. Dave CV, Winterstein AG, Park H, Cook RL, Hartzema AG. Comparative risk of lipophilic and hydrophilic statins on incident depression: a retrospective cohort study. J Affect Disord. (2018) 238:542-6. doi: 10.1016/j.jad.2018.06.021

44. Ghanizadeh A, Hedayati A. Augmentation of fluoxetine with lovastatin for treating major depressive disorder, a randomized double-blind placebo controlled-clinical trial. Depress Anxiety. (2013) 30:1084-8. doi: 10.1002/da.22195 
45. Gougol A, Zareh-Mohammadi N, Raheb S, Farokhnia M, Salimi S, Iranpour $\mathrm{N}$, et al. Simvastatin as an adjuvant therapy to fluoxetine in patients with moderate to severe major depression: A double-blind placebo-controlled trial. J Psychopharmacol. (2015) 29:575-81. doi: 10.1177/0269881115578160

46. Abbasi SH, Mohammadinejad P, Shahmansouri N, Salehiomran A, Beglar AA, Zeinoddini A, et al. Simvastatin vs. atorvastatin for improving mild to moderate depression in post-coronary artery bypass graft patients: A double-blind, placebo-controlled, randomized trial. J Affect Disord. (2015) 183:149-55. doi: 10.1016/j.jad.2015.04.049

47. Haghighi M, Khodakarami S, Jahangard L, Ahmadpanah M, Bajoghli H, Holsboer-Trachsler E, et al. In a randomized, double-blind clinical trial, adjuvant atorvastatin improved symptoms of depression and blood lipid values in patients suffering from severe major depressive disorder. J Psychiatr Res. (2014) 58:109-14. doi: 10.1016/j.jpsychires.2014.07.018

48. Molero Y, Cipriani A, Larsson H, Lichtenstein P, D’Onofrio BM, Fazel S. Associations between statin use and suicidality, depression, anxiety, and seizures: a Swedish total-population cohort study. Lancet Psychiatry. (2020) 7:982-90. doi: 10.1016/S2215-0366(20)30311-4

49. Chan D, Binks S, Nicholas JM, Frost C, Cardoso MJ, Ourselin S, et al. Effect of high-dose simvastatin on cognitive, neuropsychiatric, and health-related quality-of-life measures in secondary progressive multiple sclerosis: secondary analyses from the MS-STAT randomised, placebo-controlled trial. Lancet Neurol. (2017) 16:591-600. doi: 10.1016/S1474-4422(17)30113-8

50. Tang B, Yuan S, Xiong Y, He Q, Larsson SC. Major depressive disorder and cardiometabolic diseases: a bidirectional Mendelian randomisation study. Diabetologia. (2020) 63:1305-11. doi: 10.1007/s00125-020-05131-6

51. Luijendijk HJ, Tiemeier $H$, van den Berg JF, Bleumink GS, Hofman A, Stricker BH. Heart failure and incident late-life depression. J Am Geriatr Soc. (2010) 58:1441-8. doi: 10.1111/j.1532-5415.2010.02 921.x

Conflict of Interest: The authors declare that the research was conducted in the absence of any commercial or financial relationships that could be construed as a potential conflict of interest.

Copyright (c) 2021 Leutner, Matzhold, Kautzky, Kaleta, Thurner, Klimek and Kautzky-Willer. This is an open-access article distributed under the terms of the Creative Commons Attribution License (CC BY). The use, distribution or reproduction in other forums is permitted, provided the original author(s) and the copyright owner(s) are credited and that the original publication in this journal is cited, in accordance with accepted academic practice. No use, distribution or reproduction is permitted which does not comply with these terms. 